\title{
Mémoire et fonctions exécutives : nouvelles pistes de recherche
}

\author{
Fabienne Collette ${ }^{1,2,3}$, Lucie Angel ${ }^{4}$
}

${ }^{1}$ Centre de Recherche du Cyclotron, Université de Liège, Belgique

${ }^{2}$ Département de Psychologie : Cognition et Comportement, Université de Liège, Belgique

${ }^{3}$ Fonds National de la Recherche Scientifique (FRS-FNRS), Belgique

${ }^{4}$ UMR CNRS 7295 CeRCA, Université François Rabelais de Tours, France

Adresse pour correspondance : Dr. Fabienne Collette, Cyclotron Research Center. Allée du VI août 8, Bât B30, B-4000 Liège, Belgique

Auteur correspondant: Fabienne Collette, f.collette@ulg.ac.be

Reçu le 14 octobre 2015 ; Biologie Aujourd'hui (sous presse)

Résumé : L'existence de processus communs aux différents systèmes mnésiques et au fonctionnement exécutif a été mise en évidence par des travaux menés dans les domaines de la neuroimagerie cérébrale, de l'étude des différences individuelles (principalement dans le cadre du vieillissement normal) et, dans une moindre mesure, de la neuropsychologie. Il est maintenant bien établi que le fonctionnement exécutif dépend d'un large réseau cérébral antéro-postérieur, dont certaines régions (les régions dorsolatérales et ventrolatérales moyennes, ainsi que le cortex cingulaire antérieur dorsal) sont communes non seulement à toute une série de processus exécutifs, mais également à des processus d'encodage et de récupération de l'information en mémoire épisodique et mémoire à court terme. La présence de lésions cérébrales situées dans les régions frontales a notamment pour conséquence d'altérer l'organisation stratégique de l'information à traiter (un processus exécutif), ce qui se répercute directement sur les capacités mnésiques des patients fronto-lésés. De plus, même chez des personnes exemptes de toute pathologie cérébrale, l'efficacité du fonctionnement exécutif va non seulement se répercuter sur la performance mnésique mais également moduler les réseaux cérébraux sous-jacents. Ces données témoignent donc de l'importance de relations entre processus exécutifs et mnésiques pour un fonctionnement cognitif optimal. Les développements récents en imagerie cérébrale et électrophysiologie devraient nous permettre de mieux déterminer et comprendre le mode d'action de ces relations.

Mots clés : Mémoire épisodique / fonctions exécutives / mémoire de travail / imagerie fonctionnelle / lésions frontales

\section{Abstract : Memory processes and executive functioning : novel trends for research}

The existence of common processes to memory systems and executive functioning was evidenced by studies in the domain of cerebral neuroimaging, individual differences (mainly in normal aging) and, to a lesser extent, neuropsychology. Executive functioning depends on a large antero-posterior brain network, some regions of which (the middle dorsolateral and ventrolateral cortex, the dorsal anterior cingulate cortex) are involved in a series of executive processes, but also in encoding and retrieval of information in episodic memory and shortterm memory. A consequence of lesions in frontal areas is to impair strategical organization of the information to-be-processed (an executive process) and thus leads to a lower memory 
capacity in frontal patients. Moreover, executive abilities will influence both memory efficiency and the associated brain networks even in people without brain pathology. These data attest to the importance of the relationships between executive and memory processes for an optimal cognitive functioning. Recent advances in neuroimaging and electrophysiology data acquisition and analysis techniques should allow us to better determine and understand the fashion in which these relationships work.

Keywords : Episodic memory / executive functions / working memory / functional imaging /frontal lesions

Titre courant: Mémoire et fonctions exécutives

Le fonctionnement exécutif est classiquement défini comme un ensemble de processus cognitifs dont le rôle principal est de faciliter l'adaptation du sujet aux situations nouvelles et/ou complexes, en particulier lorsque les habiletés cognitives sur-apprises ne sont plus suffisantes. En ce sens, les fonctions exécutives ne sont liées à aucun domaine cognitif en particulier mais apparaissent plutôt avoir un rôle métacognitif, de supervision ou de contrôle sur l'ensemble des autres domaines cognitifs (perception, langage, mémoire,...). Au niveau cérébral, cela peut se traduire par le fait que les régions associées au fonctionnement exécutif vont synchroniser l'activité des régions sous-tendant les processus associés aux autres domaines de la cognition. Au niveau mnésique, la première formalisation claire des interrelations entre mémoire et fonctionnement exécutif a eu lieu dans le contexte du modèle de mémoire de travail de Baddeley $(1986,2000)$. La mémoire de travail (ou mémoire à court terme) représente notre capacité à maintenir une information active en mémoire durant un bref laps de temps après sa présentation. Dans le modèle de mémoire de travail, les systèmes passifs de stockage temporaire de l'information (boucle phonologique et registre visuospatial, représentant la mémoire à court terme) sont sous la supervision d'un administrateur central, considéré comme un système attentionnel de contrôle amodal, qui est responsable de la manipulation de l'information maintenue dans les systèmes de stockage, de la sélection de stratégies cognitives et de la coordination des informations en provenance de différentes sources. Les processus initialement attribués à l'administrateur central ont par la suite été étendus à d'autres processus de contrôle (inhibition, flexibilité, mise en place de stratégies,...) et regroupés sous le terme générique de fonctionnement exécutif. Au niveau de la mémoire épisodique (un système de mémoire à long terme permettant de se souvenir et de prendre conscience d'événements personnellement vécus dans un contexte spatial et temporal bien spécifique), il a également été montré que les processus exécutifs interviennent lors de différentes opérations d'encodage et de récupération de l'information. L'implication des processus exécutifs dans la mémoire épisodique a été conceptualisée dans le modèle neuropsychologique de Moscovitch et Winocur (1992). Ce modèle postule que deux systèmes neurocognitifs seraient impliqués dans la mémoire épisodique : 1) un système hippocampique, responsable des processus de mémoire au sens strict, aurait pour rôle d'intégrer les différentes informations qui lui parviennent en une trace mnésique à long terme, grâce à des interactions avec les régions corticales ; 2) un système de contrôle frontal qui n'interviendrait pas directement dans la mémoire mais en faciliterait le fonctionnement par la mise en place de stratégies adaptées. Les opérations gérées par cette composante sont donc considérées comme des processus qui travaillent avec la mémoire («working-with-memory processes »). De même, les premières étapes d'un apprentissage en mémoire procédurale (impliquée dans l'apprentissage d'habiletés perceptivo-motrices et cognitives) sont également sous la dépendance de certains de ces processus de contrôle (Beaunieux et al., 2006). 
Dans ce chapitre, nous détaillerons les données existantes montrant l'implication constante du fonctionnement exécutif dans les processus mnésiques, en nous centrant sur la mémoire à court terme (ou mémoire de travail) et la mémoire épisodique.

\section{Le fonctionnement exécutif : une série de processus distincts dépendants d'un réseau cérébral largement distribué.}

Les travaux en neuropsychologie et psychologie cognitive qui se sont intéressés à la structure du fonctionnement exécutif ont mis en évidence que ce terme générique regroupe en réalité un ensemble de processus variés, dont les plus fréquemment décrits sont les capacités de planification et de résolution de problèmes, la flexibilité, la mise à jour, l'inhibition et la coordination des tâches doubles. Par exemple, il a été montré, chez des sujets jeunes, que les processus exécutifs de mise à jour, flexibilité et inhibition, bien que partageant une base commune, sont clairement dissociables au niveau cognitif (Miyake et al., 2000). De même, l'observation de patients porteurs de lésions cérébrales a mis en évidence l'atteinte sélective de certains processus : certains patients présentent de bonnes performances lors d'une épreuve d'abstraction de règles (le test de Brixton), mais faibles à une tâche nécessitant d'inhiber une réponse dominante (le test de Hayling), tandis que d'autres patients présentent le profil inverse (Shallice, 1988; Shallice \& Burgess, 1991), ou encore présentent un déficit spécifique dans des situations de double tâche (Van der Linden et al., 1992).

Initialement, le fonctionnement exécutif a été considéré comme sous-tendu exclusivement par l'activité des lobes frontaux. En effet, de nombreux patients ont été décrits comme montrant une atteinte sélective de certaines de ces fonctions à la suite d'une lésion située au niveau des régions frontales (pour une présentation de ces travaux sur patients cérébro-lésés, voir Meulemans et al., 2004). Avec l'avènement des techniques modernes d'imagerie cérébrale (tomographie à émission de positons [TEP] et imagerie par résonnance magnétique fonctionnelle [IRMf]), il est toutefois apparu que le fonctionnement exécutif était sous-tendu par un réseau cérébral largement distribué, impliquant tant les régions frontales que les régions pariétales, temporales et les structures sous-corticales. Au niveau du cortex frontal, les régions les plus fréquemment rapportées sont le cortex préfrontal dorsolatéral, le cortex préfrontal ventrolatéral et le cortex cingulaire antérieur dorsal. Au sein de ce réseau, certaines régions apparaissent assez spécifiques à un processus particulier, tandis que d'autres interviennent quel que soit le processus exact mis en jeu (le «Superordinate Cognitive Control Network»; Niendam et al., 2012). De façon générale, les travaux récents ont souligné l'aspect interactif et fortement modulable du fonctionnement exécutif au niveau cérébral (voir par exemple Collette \& Salmon, 2014a).

\section{Les relations entre mémoire et fonctionnement exécutif : les données de la neuroimagerie}

En 1999, Wagner s'est interrogé sur la contribution des processus exécutifs dépendant de l'administrateur central de mémoire de travail aux processus d'encodage et de récupération de l'information en mémoire épisodique. Au moyen d'une liste de questions, il a montré que les mêmes régions préfrontales contribuent au fonctionnement de ces aspects exécutifs de la mémoire de travail et de la mémoire épisodique. De plus, de façon particulièrement intéressante, certaines de ces régions sont apparues indépendantes du type de matériel à traiter. Ainsi, les régions dorsolatérales droites (aires de Brodmann (BA) 9 et 46) et le cortex préfrontal antérieur (BA 10) qui s'activent lors de la récupération d'informations en mémoire épisodique prendraient également en charge le monitoring et la manipulation des contenus en mémoire épisodique (voir également Wagner et al.,1998). Pour rappel, cette notion de monitoring/manipulation renvoie à un des processus attribués à l'administrateur central de la mémoire de travail. Afin de valider cette interprétation de processus communs à la mémoire 
épisodique et à la mémoire de travail, Cabeza et ses collaborateurs (2002b) ont directement comparé, au moyen d'un paradigme événementiel en IRMf, l'activité cérébrale associée à la récupération d'informations résidant soit en mémoire épisodique (au moyen d'une épreuve de reconnaissance de mots) soit en mémoire de travail (au moyen d'un paradigme de réponse différé). Les résultats ont mis en évidence un réseau cérébral commun aux deux types de mémoire impliquant des régions frontales, pariétales et cérébelleuses, mais également des régions spécifiques à chaque type de mémoire. Ainsi, au niveau du cortex préfrontal, les régions dorsolatérales gauches sont recrutées par les deux types de mémoire, et reflèteraient (comme initialement proposé par Wagner) des processus de monitoring. Les régions ventrolatérales et antérieures bilatérales sont quant à elles associées de façon plus spécifique aux processus de récupération épisodique ; elles refléteraient l'intervention d'un mode de récupération ainsi que des opérations de récupération sur base d'indiçage. Par contre, les régions frontales postéro-ventrales et postéro-dorsales gauches sont plus fortement associées aux processus de mémoire de travail, reflétant probablement des opérations phonologiques ou d'autres opérations spécifiques à ce système.

Par ailleurs, il a été montré que, au sein même de la mémoire à court terme, varier la complexité des tâches induit une activité différente au sein des régions frontales. Ainsi, Owen et al. (2000) ont mis en évidence une contribution différente du cortex frontal latéral selon le recours plus ou moins important aux processus exécutifs lors d'une tâche d'empan mnésique (empan direct $v s$ empan inverse respectivement). L'empan mnésique correspond à la quantité maximum d'information qu'une personne peut stocker en mémoire à court terme à la suite d' une présentation unique du matériel (par exemple, des listes de chiffres) afin de rappeler cette information soit dans l'ordre de présentation (empan direct) soit dans l'ordre inverse de présentation (empan inverse). Durant la tâche d'empan direct, les modifications d'activité cérébrale sont observées dans le cortex ventrolatéral moyen, mais pas dans le cortex dorsolatéral moyen; durant les tâches d'empan inverse, l'augmentation d'activité cérébrale est observée dans ces deux régions (pour des résultats similaires avec des tâches d'empan verbal visuel voir Owen et al., 1996). Owen et ses collaborateurs ont donc suggéré que le cortex frontal dorsolatéral moyen sera recruté lorsqu'une manipulation active et/ou le monitoring de l'information est requis. Par contre, le cortex ventrolatéral interviendra lorsqu'un appariement relativement direct des stimuli présentés aux réponses à fournir est suffisant, comme par exemple lors des tâches d'empan direct. De plus, prenant en compte le fait que les processus de mémoire épisodique recrutent certaines régions préfrontales qui ont été associées à différents processus en mémoire à court terme, ces auteurs suggèrent qu'une approche fructueuse pourrait être de considérer que les différents processus impliqués tant en mémoire à court terme que dans les processus d'encodage et de récupération en mémoire à long terme, et dans différents processus exécutifs, dépendent tous d'un ensemble unique de composantes sous-jacentes, ces composantes intervenant de façon spécifique à chacune des tâches. Ainsi, une fonction générale du cortex frontal ventrolatéral dans le fonctionnement mnésique serait d'induire la mise en place de stratégies actives de bas niveau (par exemple la récapitulation articulatoire) et d'initier la récupération explicite de l'information stockée en mémoire à long terme. Au contraire, le cortex préfrontal dorsolatéral interviendrait dans des situations mnésiques (telles que le rappel libre) qui requièrent le monitoring à la fois des réponses produites et de l'information précédemment traitée (voir notamment Owen, 2000 ; Owen et al., 2000).

Il a également été montré qu'une fonction exécutive particulièrement importante, l'inhibition, pouvait être impliquée dans les processus mnésiques. Plus précisément, lorsque des processus inhibiteurs sont requis en mémoire épisodique, ce sera par le biais de régions cérébrales clairement associées aux processus exécutifs de suppression (notamment le cortex cingulaire 
antérieur, le gyrus frontal inférieur et le cortex orbitofrontal (Collette et al., 2006; Aron et al., 2014 ; Collette \& Salmon, 2014a). Par exemple, Schnider, Treyer \& Buck (2000) ont déterminé les régions cérébrales intervenant dans l'inhibition active de représentations mnésiques qui sont devenues non pertinentes dans le décours de la tâche. Ces auteurs ont administré une tâche de reconnaissance continue dans laquelle des images étaient présentées, lors de séries successives, aux participants, ces derniers devant indiquer quelles images avaient déjà été présentées, mais dans la série en cours uniquement. Une performance correcte nécessitait donc de faire la distinction entre l'essai en cours et les essais précédents. Cette capacité de distinction «inter-essais »a été associée à une activité spécifique dans la région orbitofrontale postérieure. Par conséquent, les auteurs ont attribué à cette région un rôle d'inhibition des informations mnésiques devenues non pertinentes dans le contexte de la tâche en cours.

Dans une étude plus récente, Chiu et Egner (2015a ; voir également Chiu \& Egner, 2015b) ont montré que, lors de l'encodage incident d'informations, fournir une réponse motrice par rapport à inhiber cette réponse (par exemple, appuyez sur la touche si le visage est masculin, mais ne donnez pas de réponse s'il est féminin) amenait à de meilleures performances comportementales. De plus, l'activité du cortex préfrontal ventrolatéral, une région précédemment associée à l'encodage en mémoire épisodique, et plus particulièrement à des processus de sélection qui dirigent l'attention vers l'information pertinente en fonction des objectifs de la tâche (Milner et al., 1985 ; Miller \& Cohen, 2001 ; Blumenfeld \& Ranganath 2007), était associée (1) de façon négative à la demande en ressource nécessaire à une inhibition correcte, et (2) de façon positive à la reconnaissance correcte des informations préalablement encodées. Ce pattern de résultats indique qu'à la fois l'inhibition d'une réponse motrice et l'encodage incident en mémoire épisodique partagent des ressources neurales, et que devoir inhiber une réponse motrice concomitante à la présentation d'un item va détourner temporairement les ressources attentionnelles du traitement de cet item.

De façon plus générale, McNab et al. (2008) ont montré la présence de patterns d'activation communs entre trois tâches d'inhibition (stop-task, go/no-go et flanker) et deux tâches de mémoire à court terme (paradigme de Sternberg pour de l'information verbale et spatiale). Les régions activées de façon commune lors de la réalisation de ces cinq tâches se situent au niveau du gyrus frontal inférieur droit, du gyrus frontal moyen droit et des régions pariétales droites. Ces résultats confortent l'idée selon laquelle les tâches de mémoire de travail et d'inhibition ont des soubassements neuraux communs, qui constitueraient la base des interrelations entre ces systèmes. Dans ce contexte, Duncan et Owen (2000) ont proposé qu'un réseau frontal spécifique, incluant les régions dorsolatérales et ventrolatérales moyennes, ainsi que le cortex cingulaire antérieur dorsal, soit associé de façon consistante avec un ensemble varié de tâches nécessitant des demandes cognitives diverses (telles que des aspects perceptifs, de sélection de réponse, de mémoire de travail, de mémoire épisodique et de résolution de problèmes; ces tâches incluant, entre autres processus, un recours au fonctionnement exécutif), tandis que le reste du cortex frontal, incluant la plupart des régions médiales et orbitales, est largement insensible aux demandes de ces tâches. Parmi ce réseau, Owen et ses collaborateurs (Owen, 2000; Owen et al., 2000) ont attribué à la région préfrontale dorsolatérale (BA 9/46) un rôle de contrôle ("monitoring ») des réponses fournies et de l'information présentée précédemment. La région cingulaire a, quant à elle, été associée à des processus de résolution des conflits et de gestion de la compétition entre différentes régions cérébrales impliquées dans une tâche cognitive (Bush et al., 2000). Finalement, Derrfuss et al. (2004) ont proposé que la jonction frontale inférieure soit impliquée dans la mise à jour des représentations nécessaires à la réalisation d'une tâche, en fournissant un 
signal qui modifie les appariements stimulus-réponse préalablement activés dans les régions postérieures.

\section{Les relations en mémoire et fonctionnement exécutif: les données de la neuropsychologie}

À partir des années 80, l'existence de troubles de la mémoire épisodique consécutifs à une lésion frontale a été progressivement attestée par un nombre croissant d'études (pour une présentation, voir Van der Linden et al., 1999). Toutefois, ces études ne s'accordent pas quant au rôle précis joué par les structures frontales dans le fonctionnement mnésique. Dans une méta-analyse réalisée par Wheeler et al. (1995), il apparaît que la présence de lésions frontales est plus souvent associée à des déficits dans des tâches de rappel libre (80\%) que dans des tâches de rappel indicé $(50 \%)$ et de reconnaissance $(8 \%)$. Une analyse plus approfondie de ces données indique que les régions frontales interviendraient préférentiellement lorsque la tâche (principalement de rappel, mais également des épreuves de reconnaissance de nature plus stratégique) nécessite la mise en place de stratégies d'organisation lors de la phase d'encodage, et dans la recherche active ou le contrôle (monitoring) de la réponse lors de la phase de récupération. En effet, de nombreux auteurs (voir par exemple Parkin et al., 1994 ; Stuss et al., 1994 ; Gershberg \& Shimamura, 1995) ont observé une mauvaise utilisation des stratégies d'organisation de l'information lors d'épreuves de rappel, ces aspects d'organisation stratégique relevant du fonctionnement exécutif.

Ces données montrent donc également, bien que de façon plus indirecte, l'importance de l'intégrité des processus stratégiques, dépendant des régions frontales, dans la mise en œuvre de processus efficaces en mémoire épisodique. Il n'existe toutefois pas d'études, à notre connaissance, qui aient directement comparé les performances mnésiques de patients frontaux porteurs ou non d'un syndrome dysexécutif (ou présentant une atteinte exécutive plus ou moins marquée). Ce type d'étude a par contre été réalisé dans le contexte de travaux sur les changements cognitifs associés au vieillissement normal.

\section{Les relations entre mémoire et fonctionnement exécutif : l'exemple de la variabilité inter-individuelle au cours du vieillissement normal}

L'idée selon laquelle les fonctions exécutives jouent un rôle clé dans le fonctionnement optimal de la mémoire a incité les chercheurs à explorer leur rôle dans certains dysfonctionnements mnésiques, notamment ceux observés au cours du vieillissement normal. Ainsi, une hypothèse dite " exécutive " s'est imposée comme un des principaux modèles explicatifs du vieillissement neurocognitif. Cette hypothèse suggère que l'altération des régions frontales qui sous-tendent les fonctions exécutives expliquerait les déficits cognitifs qui s'observent au cours du vieillissement dans les tâches cognitives où les fonctions exécutives sont fortement impliquées (Moscovitch \& Winocur, 1992; West, 1996 ; Raz, 2000).

Cette idée s'appuie sur un faisceau d'éléments neurobiologiques et neuropsychologiques convergents. Un certain nombre d'observations indiquent que les modifications neurobiologiques (volume cérébral, activité au repos, fonctionnement neurochimique) au cours du vieillissement sont plus précoces et plus marquées au niveau des régions préfrontales (West, 1996 ; Raz, 2000 ; Buckner, 2004; Dennis \& Cabeza, 2008). De plus, les personnes âgées présentent des déficits importants dans les tests évaluant le fonctionnement exécutif (voir pour revue Collette \& Salmon, 2014b). D'autre part, l'hypothèse exécutivo-frontale repose également sur le constat de la similarité qualitative du profil mnésique des personnes âgées avec celui de patients cérébro-lésés frontaux (Moscovitch \& Winocur, 1992). 
L'implication du système exécutivo-frontal dans la mise en place de stratégies mnésiques par les personnes âgées a été confirmée par différents travaux. Ainsi, le déclin avec l'âge de la performance dans diverses tâches épisodiques, en particulier dans des tâches très stratégiques, serait fortement médiatisé par le fonctionnement exécutif (Troyer et al., 1994 ; Parkin, 1997; Salthouse et al., 2003), même lorsque ce dernier est mis en concurrence avec d'autres facteurs (Crawford et al., 2000). Dans une étude particulièrement intéressante réalisée en 1995, Glisky, Polster et Routhieaux ont réparti des participants âgés sur la base d'une mesure composite reflétant le fonctionnement exécutif et ont comparé leurs performances à deux épreuves de mémoire épisodique : une épreuve de mémoire de l'item (reconnaissance des phrases) et une épreuve de mémoire de la source (reconnaissance de la voix ayant prononcé la phrase). Les résultats indiquent que les deux groupes ne diffèrent pas pour la mémoire de l'item mais bien pour la mémoire de source, ce qui pourrait indiquer que de plus faibles capacités exécutives ne permettent pas de construire une représentation mnésique riche et composite, intégrant les multiples traits indépendants (et parfois périphériques, comme ici la voix par rapport au contenu sémantique de la phrase) des expériences sensorielles. Bugaiska et al. (2007) ont également confirmé que la réduction du nombre de réponses de type Remember, reflétant une récupération épisodique riche incluant des détails contextuels associés à l'item, chez les sujets âgés, était spécifiquement corrélée aux capacités exécutives.

D'autre part, plusieurs études récentes (Glisky \& Kong, 2008 ; Bouazzaoui et al., 2012) ont pu mettre en évidence que les adultes âgés engageraient davantage que des jeunes les fonctions exécutives pour réaliser des tâches de mémoire épisodique. Les performances de mémoire épisodique sont systématiquement significativement corrélées chez les adultes âgés avec les performances à des tests exécutifs, alors que ces corrélations n'apparaissent pas chez les jeunes adultes. Ce recours plus important avec l'âge aux ressources exécutives permettrait de réduire les différences jeunes-âgés (Bouazzaoui et al., 2012). Ces observations comportementales sont cohérentes avec les données récentes de la neuroimagerie qui montrent que les sujets âgés présentent parfois lors de la réalisation de tâches de mémoire des activations supplémentaires par rapport aux sujets jeunes dans des régions impliquées dans le réseau exécutif (Park \& Reuter-Lorenz, 2009) et que ce surcroît d'activation pourrait être compensatoire (Cabeza et al., 2002a). Ce plus grand engagement du contrôle exécutif chez les adultes âgés peut sembler paradoxal dans la mesure où les ressources exécutives, comme nous l'avons rapporté plus haut, déclinent fortement au cours du vieillissement (Collette \& Salmon, 2014b). En d'autres termes, les fonctions exécutives seraient avec l'âge à la fois une source de dysfonctionnement et de compensation en mémoire (Greenwood \& Parasuraman, 2010 ; Bouazzaoui et al., 2012; Craik \& Rose, 2012), et cela en lien avec les caractéristiques exactes à la fois des participants et de la tâche (Angel et al., 2010, 2015).

D'autres arguments mettant en lien la mémoire et le fonctionnement exécutif au cours du vieillissement proviennent d'études en imagerie cérébrale. Des travaux suggèrent en effet un lien entre la variabilité inter-individuelle dans les capacités exécutives et les modifications des patterns d'activation cérébrale au cours de tâches de mémoire. Une étude utilisant la méthode des potentiels évoqués a démontré que les différences liées à l'âge au niveau de l'effet « old/new ", un indicateur électrophysiologique des processus de récupération épisodique, étaient modulées par le niveau de fonctionnement exécutif des participants (Angel et al., 2010). Une autre étude, en IRMf cette fois, a permis de mettre en évidence le fait que les réseaux cérébraux activés par des participants âgés vont varier en fonction de leurs capacités exécutives lors d'une tâche de mémoire épisodique possédant deux niveaux de difficulté (le niveau de difficulté dépendant du nombre de présentations (1 vs 2) des items lors de l'encodage) (Angel et al., 2015). Plus précisément, le groupe de participants présentant un niveau exécutif élevé active davantage que les participants âgés avec un niveau exécutif plus 
faible et que des participants jeunes certaines régions cérébrales, en particulier au niveau frontal et pariétal droit, dans la condition la plus facile mais surtout dans la condition la plus difficile, ce qui pourrait les avoir aidés à compenser leurs difficultés et à maintenir un haut niveau de performance mnésique, même dans la condition la plus difficile.

Une autre étude en IRMf, menée cette fois seulement sur des sujets jeunes sains, complète de façon intéressante ces derniers résultats, en montrant que cette modulation des réseaux cérébraux liés aux processus mnésiques par les capacités exécutives serait un processus très général, et non une conséquence des modifications cognitives associées à l'avancée en âge. Minamoto, Osaka et Osaka (2010) ont en effet comparé les patterns d'activité cérébrale lors d'une tâche de mémoire à court terme avec distraction chez des participants jeunes présentant des capacités exécutives faibles ou élevées. La tâche de mémoire à court terme consistait en la présentation successive de trois visages que les sujets devaient mémoriser afin de déterminer, après un intervalle de rétention, si l'un d'entre eux correspondait au visage cible. Durant l'intervalle de rétention, trois autres visages ou trois stimuli brouillés étaient brièvement présentées, la présentation des visages devant être inhibée afin de ne pas interférer avec le maintien des informations cibles. Les données comportementales montrent une meilleure performance à cette tâche pour les participants avec les capacités en mémoire de travail les plus élevées. Cette meilleure performance comportementale est associée à une activité plus importante au niveau du gyrus frontal moyen gauche ainsi qu'à une diminution d'activité au niveau des régions fusiformes bilatérales (associées au traitement des visages). Ces résultats indiquent que les meilleures capacités d'inhibition chez les participants avec de fortes capacités exécutives pourraient dépendre d'une modulation top-down des régions frontales moyennes sur les régions perceptives postérieures.

\section{Conclusion}

Les travaux présentés dans cette revue montrent donc clairement les relations existant entre fonctionnement mnésique et exécutif. Nous avons ainsi vu que tant la mémoire épisodique que la mémoire à court et les processus exécutifs ont des substrats cérébraux en commun. Une des caractéristiques de ces substrats communs est qu'ils semblent sous-tendre des processus de nature plutôt stratégique, tels que le monitoring de l'information à encoder et/ou à récupérer, le monitoring des réponses produites, ou encore la nécessité d'inhiber certaines informations ou processus qui viennent interférer avec la tâche en cours. Dans une perspective neuropsychologique, il apparaît que la présence de lésions dans des régions dédiées au fonctionnement exécutif influence directement les capacités mnésiques. De plus, même en l'absence de lésions cérébrales, la variabilité individuelle dans l'efficacité exécutive va se répercuter sur les performances en mémoire épisodique, et surtout sur le recrutement des régions cérébrales lors de la réalisation de ces tâches.

Afin d'améliorer notre compréhension des processus mnésiques, les travaux futurs devront donc prendre explicitement en compte cette influence exécutive, et surtout déterminer si la dynamique observée à ce jour dans le cadre du vieillissement normal se retrouve dans d'autres populations, telles que des patients avec lésions cérébrales acquises (que ces dernières soient focales ou diffuses) ou des enfants à différents stades de développement. Au niveau de la neuroimagerie, si les études à ce jour ont permis d'identifier les régions communes à ces différents processus, il apparaît indispensable dans le futur d'explorer à la fois les interactions au sein de ces régions ainsi qu'avec les régions postérieures impliquées dans le fonctionnement de la mémoire épisodique et de la mémoire à court terme pour différents types de matériel (mots, images, visages,...). Les développements récents dans les techniques d'acquisition et d'analyse de données en imagerie cérébrale et (notamment celles en lien avec la connectivité entre régions, tant dans une perspective fonctionnelle que structurelle) et en 
électrophysiologie devraient permettre des avancées rapides et significatives de nos connaissances sur les relations entre fonctionnement exécutif et efficacité mnésique.

\section{Références}

Angel, L., Fay, S., Bouazzaoui, B., and Isingrini, M. (2010). Individual differences in frontal functioning modulate age effects on the ERP correlates of retrieval success. Neuropsychologia, 48, 3540-3553.

Angel, L., Bastin, C., Genon, S., E., S., Fay, S., Balteau, E., Maquet, P., Luxen, A., Isingrini, M., and F., C. (2015). Neural correlates of successful memory retrieval in aging: Do executive functioning and task difficulty matter? Brain Res, In press.

Aron, A.R., Robbins, T.W., and Poldrack, R.A. (2014). Inhibition and the right inferior frontal cortex: one decade on. Trends Cogn Sci, 18, 177-185.

Baddeley, A. (2000). Short-term and working memory. In The Oxford Handbook of Memory, E. Tulving, and F.I.M. Craick, (Eds.), Oxford University Press, Oxford, pp. 77-92.

Baddeley, A.D. (1986). Working memory. Clarendon Press, Oxford.

Beaunieux, H., Hubert, V., Witkowski, T., Pitel, A., Rossi, S., Danion, J.M., Desgranges, B., and Eustache, F. (2006). Which processes are involved in cognitive procedural learning? Memory, 14, 521-539.

Blumenfeld, R.S., and Ranganath, C. (2007). Prefrontal cortex and long-term memory encoding: an integrative review of findings from neuropsychology and neuroimaging. Neuroscientist, 13, 280-291.

Bouazzaoui, B., Angel, L., Fay, S., Taconnat, L., Froger, C., and Isingrini, M. (2012). Does the greater involvement of executive control in memory with age act as a compensatory mechanism? Can J Exp Psychol, 68, 59-66.

Buckner, R.L. (2004). Memory and executive function in aging and AD: Multiple factors that cause decline and reserve factors that compensate. Neuron, 44, 195-208.

Bugaiska, A., Clarys, D., Jarry, C., Taconnat, L., Tapia, G., Vanneste, S., and Isingrini, M. (2007). The effect of aging in recollective experience: the processing speed and executive functioning hypothesis. Conscious Cogn, 16, 797-808.

Bush, G., Luu, P., and Posner, M.I. (2000). Cognitive and emotional influences in anterior cingulate cortex. Trends Cogn Sci, 4, 215-222.

Cabeza, R., Anderson, N.D., Locantore, J.K., and McIntosh, A.R. (2002a). Aging gracefully: Compensatory brain activity in high-performing older adults. Neuroimage, 17, 13941402.

Cabeza, R., Dolcos, F., Graham, R., and Nyberg, L. (2002b). Similarities and differences in the neural correlates of episodic memory retrieval and working memory. Neuroimage, 16, 317-330.

Chiu, Y.C., and Egner, T. (2015a). Inhibition-Induced Forgetting Results from Resource Competition between Response Inhibition and Memory Encoding Processes. J Neurosci, 35, 11936-11945.

Chiu, Y.C., and Egner, T. (2015b). Inhibition-induced forgetting: when more control leads to less memory. Psychol Sci, 26, 27-38.

Collette, F., Hogge, M., Salmon, E., and Van der Linden, M. (2006). Exploration of the neural substrates of executive functioning by functional neuroimaging. Neuroscience, 139, 209-221.

Collette, F., and Salmon, E. (2014a). Fonctionnement exécutif et réseaux cérébraux. Revue de Neuropsychologie, 6, 256-266.

Collette, F., and Salmon, E. (2014b). Les modifications du fonctionnement exécutif dans le vieillissement normal. Psychologie Française, 59, 41-58. 
Craik, F.I., and Rose, N.S. (2012). Memory encoding and aging: a neurocognitive perspective. Neurosci Biobehav Rev, 36, 1729-1739.

Crawford, J.R., Bryan, J., Luszcz, M.A., Obonsawin, M.C., and Stewart, L. (2000). The executive decline hypothesis of cognitive aging: Do executive deficits qualify as differential deficits and do they mediate age-related memory decline? Aging, Neuropsychology and Cognition, 7, 9-31.

Dennis, N.A., and Cabeza, R. (2008). Neuroimaging of healthy cognitive aging. In The handbook of aging and cognition, Third edition, F.I.M. Craik, and T.A. Salthouse, (Eds.), Psychology Press, New York, pp. 1-54.

Derrfuss, J., Brass, M., and Yves von Cramon, D. (2004). Cognitive control in the posterior frontolateral cortex: evidence from common activations in task coordination, interference control, and working memory. Neuroimage, 23, 604-612.

Duncan, J., and Owen, A.M. (2000). Common regions of the human frontal lobe recruited by diverse cognitive demands. Trends Neurosci, 23, 475-483.

Gershberg, F.B., and Shimamura, A.P. (1995). Impaired use of organizational strategies in free recall following frontal lobe damage. Neuropsychologia, 13, 1305-1333.

Glisky, E.L., and Kong, L.L. (2008). Do young and older adults rely on different processes in source memory tasks? A neuropsychological study. J Exp Psychol Learn Mem Cogn, 34, 809-822.

Glisky, E.L., Polster, M.R., and Routhieaux, B.C. (1995). Double dissociation between item and source memory. Neuropsychology, 9, 229-239.

Greenwood, P.M., and Parasuraman, R. (2010). Neuronal and cognitive plasticity: a neurocognitive framework for ameliorating cognitive aging. Front Aging Neurosci, 2, 150.

McNab, F., Leroux, G., Strand, F., Thorell, L., Bergman, S., and Klingberg, T. (2008). Common and unique components of inhibition and working memory: an fMRI, withinsubjects investigation. Neuropsychologia, 46, 2668-2682.

Meulemans, T., Collette, F., and Van der Linden, M. (2004). Neuropsychologie des fonctions exécutives. Solal, Marseille.

Miller, E.K., and Cohen, J.D. (2001). An integrative theory of prefrontal cortex function. Annu Rev Neurosci, 24, 167-202.

Milner, B., Petrides, M., and Smith, M.L. (1985). Frontal lobes and the temporal organization of memory. Hum Neurobiol, 4, 137-142.

Minamoto, T., Osaka, M., and Osaka, N. (2010). Individual differences in working memory capacity and distractor processing: possible contribution of top-down inhibitory control. Brain Res, 1335, 63-73.

Miyake, A., Friedman, N.P., Emerson, M.J., Witzki, A.H., and Howerter, A. (2000). The unity and diversity of executive functions and their contribution to complex "frontal lobe" tasks: A latent variable analysis. Cogn Psychol, 41, 49-100.

Moscovitch, M., and Winocur, G. (1992). The neuropsychology of memory and aging. In The handbook of Aging and Cognition, F.I.M. Craik, and T.A. Salthouse, (Eds.). Lawrence Erlbaum Associates, Publishers, Hillsdale, New Jersey.

Niendam, T.A., Laird, A.R., Ray, K.L., Dean, Y.M., Glahn, D.C., and Carter, C.S. (2012). Meta-analytic evidence for a superordinate cognitive control network subserving diverse executive functions. Cogn Affect Behav Neurosci, 12, 241-268.

Owen, A.M. (2000). The role of the lateral frontal cortex in mnemonic processing: The contribution of functional neuroimaging. Exp Brain Res, 133, 33-43.

Owen, A.M., Evans, A.C., and Petrides, M. (1996). Evidence for a two-stage model of spatial working memory processing within the lateral frontal cortex: a positon emission tomography study. Cereb Cortex, 6, 31-38. 
Owen, A.M., Lee, A.C., and Williams, E.J. (2000). Dissociating aspects of verbal working memory within the human frontal lobes: Further evidence for a "process-specific" model of lateral frontal organisation. Psychobiology, 28, 146-155.

Park, D.C., and Reuter-Lorenz, P. (2009). The adaptive brain: aging and neurocognitive scaffolding. Annu Rev Psychol, 60, 173-196.

Parkin, A.J. (1997). Normal age-related memory loss and its relation to frontal lobe dysfunction. In P. Rabbitt (Ed.), Methodology of frontal and executive function Psychology Press, Hove, UK, pp. 177-190.

Parkin, A.J., Yeomans, J., and Bindschaedler, C. (1994). Further characterization of the executive memory impairment following frontal lobe lesions. Brain Cogn, 26, 23-42.

Raz, N. (2000). Aging of the brain and its impact on cognitive performance: Integration of structural and functional findings. In Handbook of aging and cognition, F. Craick, and T. Salthouse (Eds.), Erlbaum, Hillsdale, NJ, pp. 1-90.

Salthouse, T.A., Atkinson, T.M., and Berish, D.E. (2003). Executive functioning as a potential mediator of age-related cognitive decline in normal aging. $J$ Exp Psychol Gen, 132, 566-594.

Schnider, A., Treyer, V., and Buck, A. (2000). Selection of currently relevant memories by the human posterior medial orbitofrontal cortex. J Neurosci, 20, 5880-5884.

Shallice, T. (1988). From neuropsychology to mental structures. Cambridge University Press, Cambridge.

Shallice, T., and Burgess, P.W. (1991). Deficits in strategy application following frontal lobe damage in man. Brain, 114, 727-741.

Stuss, D.T., Alexander, M.P., Palumbo, C.L., Buckle, L., Sayer, L., and Pogue, J. (1994). Organizational strategies of patients with unilateral or bilateral frontal lobe injury in word list learning tasks. Neuropsychology, 3, 355-373.

Troyer, A.K., Graves, R.E., and Cullum, C.M. (1994). Executive functioning as a mediator of the relationship between age and episodic memory in healthy aging. Aging, Neuropsychology, and Cognition, 1, 45-53.

Van der Linden, M., Andrès, P., and Marczewski, P. (1999). Le rôle des lobes frontaux dans le fonctionnement de la mémoire épisodique. In : Neuropsychologie des lobes frontaux, M. Van der Linden, X. Seron, D. Le Gall, and P. Andrès, (Eds.), Solal, Marseille, pp. 167-201.

Van der Linden, M., Coyette, F., and Seron, X. (1992). Selective impairment of the "central executive" component of working memory: A single case study. Cogn Neuropsychol, 9, 301-326.

Wagner, A.D. (1999). Working memory contributions to human learning and remembering. Neuron, 22, 19-22.

Wagner, A.D., Schacter, D.L., Rotte, M., Koutstaal, W., Maril, A., Dale, A.M., Rosen, B.R., and Buckner, R.L. (1998). Building memories: Remembering and forgetting of verbal experiences as predicted by brain activity. Science, 281, 1188-1191.

West, R.L. (1996). An application of prefrontal function theory to cognitive aging. Psychol Bull, 120, 272-292.

Wheeler, M.A., Stuss, D.T., and Tulving, E. (1995). Frontal lobe damage produces episodic memory impairment. J Int Neuropsychol Soc, 1, 525-536. 